\title{
DEL POSTO DIALETTOMETRICO CHE SPETTA AI PUNTI GALLOITALICI AIDONE, SPERLINGA E SAN FRATELLO NEL SISTEMA DELLA RETE DELL'AIS ${ }^{1}$
}

\section{OSSERVAZIONI PRELIMINARI}

I lettori di Linguistica hanno avuto modo già cinque volte di informarsi sulla dialettometria (DM) e sull'avanzamento dei suoi metodi e risultati, rispettivamente nei volumi 26 (1986), 28 (1988), 31 (1991), 40 (2000) e 46 (2006). I nostri contributi del 1986, 1988 e 1991 vertevano su argomenti geolinguistici relativi all'AIS, mentre quelli del 2000 e 2006 trattavano problemi geolinguistici relativi alla Galloromania, tanto di quella moderna (2000) quanto di quella medioevale (2006). Nel presente articolo ci occuperemo di nuovo dei dati dell'AIS approfittando in merito dell'esuberante ricchezza dei risultati ottenuti nel corso dell'analisi dialettometrica di tutti i dati dell'AIS, relativa cioè alla sua rete intera (con 407 punti di rilevamento) ed a tutti gli otto volumi atlantistici.

I lavori di dialettometrizzazione dell'AIS si sono svolti a Salisburgo dal 2005 al $2009 .^{2}$ Sono state finora pubblicate due relazioni di lavoro (2007 e 2008), corredate ambedue da molti grafici a colori ma non ancora basate sulla totalità dei dati analizzabili. Mentre la nostra relazione di lavoro del 2007 poggiava sullo spoglio di 688 carte originali dell'AIS spogliate (equivalenti a 1746 carte di lavoro [CL]), quella del 2008 era già basata su 967 carte originali dell'AIS (commutate in $3076 \mathrm{CL}$ ). La messe completa della dialettometrizzazione dell'AIS verte su 1320 carte originali dell'AIS dalle quali abbiamo tratto $3911 \mathrm{CL}$.

\section{DUE PAROLE SULLA DIALETTOMETRIA E SULLA DIALETTOMETRIZ- ZAZIONE DELL'AIS}

La DM costituisce - come si sa - un prolungamento diretto della geografia linguistica (romanza) classica rappresentata dai grandi atlanti linguistici nazionali come l'ALF (per la Galloromania) e l'AIS (per l'Italo-, Sardo- e Retoromania) nonché

\footnotetext{
*Indirizzo dell'autore: Fachbereich Romanistik, Akademiestrasse 24, A-5020 Salzburg, Austria. Email: Hans.Goebl@sbg.ac.at

${ }^{1}$ La somiglianza sintattica e semantica col titolo del ben noto articolo di G.I. Ascoli del 1876 («Del posto che spetta al ligure nel sistema dei dialetti italiani») è tutt'altro che fortuita.

2 Per ulteriori dettagli logistici e amministrativi rinvio alle informazioni date alla fine di questo contributo.
} 
dalle rispettive ulteriori interpretazioni fatte sia da parte degli autori stessi dell'ALF (Jules Gilliéron) e dell'AIS (Karl Jaberg e Jakob Jud) ${ }^{3}$ che dai loro seguaci dentro o fuori della Francia o dell'Italia.

Il termine ed il metodo della «dialectométrie» sono stati ideati nel 1973 dal geolinguista francese Jean Séguy (1914-1973). Nei successivi quattro decenni la DM si è sviluppata in maniera molto differenziata, tanto da dare l'avvio alla nascita di alcune «scuole» DM particolari ${ }^{4}$ una delle quali a Salisburgo.

La «Scuola Dialettometrica di Salisburgo» (SDMS) ${ }^{5}$ mira, tramite la considerazione sintetica della totalità dei dati di un qualsiasi atlante linguistico, allo studio della "gestione basilettale dello spazio geografico da parte dell'Homo loquens», assumendo che ci siano, accanto alle strutture geolinguistiche delle singole carte dei diversi atlanti linguistici, anche strutture geolinguistiche nascoste (o di profondità) che, alla pari di leggi linguistiche spaziali, governano le con- e divergenze dei dialetti di un determinato dominio linguistico.

A tal fine la SDMS utilizza, per la sintesi quantitativa dei dati atlantistici, i metodi della classificazione numerica ${ }^{6}$ e, per la visualizzazione sfumata dei risultati calcolati, quelli della cartografia quantitativa internazionale. L'elemento della visualizzazione appropriata dei risultati numerici è molto importante perchè rappresenta da una parte una continuazione della tradizione cartografica della geolinguistica romanza classica e dall'altra un mezzo euristico molto efficace per una buona comunicazione scientifica. ${ }^{7}$

Per una rapida e confortevole esecuzione dei calcoli DM e la loro successiva visualizzazione, la SDMS dispone da ormai dieci anni di un programma informatico molto potente chiamato VDM («Visual DialectoMetry») elaborato dal nostro amico e collaboratore Edgar Haimerl. Attualmente il programma VDM funziona in tre lingue: tedesco, italiano e catalano. I grafici di questo contributo sono stati eseguiti mediante la versione italiana di VDM e corrispondono a schermate prodotte direttamente con VDM.

Ai fini di questo articolo imperniato sul problema delle isole linguistiche galloitaliche ${ }^{8}$ della Sicilia abbiamo scelto quattro «sguardi dialettometrici (DM)» che consentono di

\footnotetext{
3 Si veda in merito, a mo' d'esempio, Jaberg (1936).

4 Cito, accanto alla SDMS, le scuole DM di Groningen (cf. Heeringa 2004 e Nerbonne/Kretzschmar 2006) e di Georgia, SUA (cf. Kretzschmar/Schneider 1996).

5 Per il lavori svolti secondo la «filosofia DM» della SDMS rinvio anche ai contributi di Roland Bauer (2003, 2005 e 2009).

${ }^{6}$ Cf. in merito i manuali classici di Bock 1974 (ted.), Chandon/Pinson 1981 (franc.) e Sneath/Sokal 1973 (ingl.)

${ }^{7}$ Per i principi elementari della DM cf. Goebl 1982 e 1984a; per una descrizione meno densa (accompagnata però sempre da molti grafici appositamente commentati) cf. i nostri contributi del 1981, 1983, 1987, 2000 e 2003a (tutti in francese) nonché del 1984b, 1988, 1991, 2007b e 2008 (in italiano) e del 2006 (in inglese).

8 Preferiamo - con S. G. Trovato (1998 e 1999) e gli autori del «Lexikon der Romanistischen Linguistik» - la forma sintetica galloitalico alla variante analitica gallo-italico, reiterando così l'esperienza precedente con il termine ascoliano franco-provenzale che sin dai lavori del noto francoprovenzalista Gaston Tuaillon viene scritto senza trattino (francoprovenzale, francoprovençal).
} 
considerare i problemi geotipologici relativi alle isole linguistiche sotto quattro angolazioni diverse. È ovvio che la DM offre ancora tante altre angolazioni interessanti, alla presentazione delle quali dobbiamo purtroppo rinunciare per motivi di spazio.

La dialettometrizzazione dell'AIS comprendeva grosso modo le tappe seguenti:

1) definizione della rete di lavoro

2) definizione della lista nonché del numero delle carte originali dell'AIS analizzabili

3) creazione della matrice dei dati tramite la «tassazione ${ }^{9} »$ delle carte dell'AIS previamente selezionate

4) inserzione dei dati tassati nel programma VDM

5) applicazione di tutte le possibilità classificatorie e visualizzatrici di VDM alla matrice dei dati testè menzionata.

Visto che per qualsiasi calcolo tassometrico la presenza di dati mancanti nella rispettiva matrice dei dati è altamente pregiudizievole, le tappe 1 e 2 della suddetta catena di lavoro devono riferirsi prevalentemente all'accertamento della più o meno grande completezza formale dei dati originali dell'AIS.

Dato che in molte località settentrionali dell'AIS è stato applicato soltanto il questionario ridotto dell'AIS e che anche in molti altri posti dell'AIS si manifestano, per vari motivi, lacune documentarie talvolta considerevoli, si è reso necessario scartare da un lato due dozzine di località settentrionali dalla nostra rete di lavoro e tralasciare dall'altro pressoché 400 delle 1705 carte originali dell'AIS. Si può dunque stabilire, ai fini del presente contributo, la tabella seguente:

\begin{tabular}{|l|c|c|}
\hline & $\begin{array}{c}\text { Stock disponibili } \\
\text { nell'AIS }\end{array}$ & $\begin{array}{c}\text { Utilizzate per la dialettometriz- } \\
\text { zazione dell'AIS }\end{array}$ \\
\hline Numero delle località-AIS & 407 & $382^{10}$ \\
\hline $\begin{array}{l}\text { Numero delle carte originali } \\
\text { dell'AIS }\end{array}$ & 1705 & 1320 \\
\hline
\end{tabular}

Dalle 1320 carte-AIS prese in considerazione abbiamo estratto, tramite l'analisi qualitativa della tassazione, $3911 \mathrm{CL}$, l'insieme delle quali costituisce la nostra matrice dei dati. Le carte 1 e 2 ne rappresentano campioni tipici. Mentre la carta 1 è il risul-

9 Per tassazione s'intende una procedura classificatoria che mira alla definizione di tipi linguistici «ideali». Si tratta di un lavoro svolto correntemente dalla quasi totalità dei protagonisti della geografia linguistica romanza classica; per un'applicazione recente di questa procedura ai dati dell'ALF cf. Brun-Trigaud/Le Berre/Le Dû (2005).

10 In questa cifra sono compresi i punti artificiali 999 (italiano standard) e 998 (francese standard), insediati nella nostra rete rispettivamente nei dintorni di Firenze e della Valle d'Aosta. Ciò non impedisce che nel nostro contributo DM del 2008 abbiamo utilizzato ancora due altre reti dell'AIS: una rete molto più fitta con 414 punti (comprendente alcune inchieste doppie fatte nelle grandi città) ed una rete abbastanza assottigliata con solo 296 punti. 
tato di una tassazione fonetica di stampo vocalico, ${ }^{11}$ la carta 2 mostra, sulla scorta di molte altre tipizzazioni fatte e pubblicate nei manuali e sulle riviste della romanistica da oltre 80 anni, la ripartizione geografica delle denominazioni italo-, sardo- e retoromanze della «nipote». La tassazione della carta 1 consta di dieci tassati fonetici e di altrettante aree geolinguistiche con dimensioni e configurazioni molto diverse tra di loro, mentre quella della carta 2 abbraccia solo sei tassati lessicali diversi con le rispettive aree di diffusione. La variabilità interna delle $3911 \mathrm{CL}$ analizzate va da 2 a 153 tassati; il numero complessivo di tutti i tassati analizzati (e quindi anche delle rispettive aree di diffusione) ammonta a 43564 unità.

Possiamo dunque dire che la «gestione basilettale dello spazio dell'AIS da parte dei locutori delle 382 località da noi esaminate» dipende dalle molteplici con- e divergenze delle 43564 aree di stampo fonetico, morfosintattico e lessicale individuate nel corso della nostra analisi tassatoria degli otto volumi atlantistici dell'AIS.

\section{L'UTILITÀ GEOLINGUISTICA DELLE ISOLE LINGUISTICHE COMPRESE NELLA RETE DELL'AIS}

Mentre Jules Gilliéron si è limitato all'inclusione di una sola isola linguistica nella rete del suo ALF, ${ }^{12}$ gli autori dell'AIS ne hanno presi in considerazione otto: oltre alle tre isole linguistiche galloitaliche situate in Sicilia (Aidone - P. 836, Sperlinga - P. 836, San Fratello - P. 817) si tratta di un'isola francoprovenzale (Faeto - P. 715), di un'isola linguistica provenzale (Guardia Piemontese - P. 760), di due isole greche (Ghorio - P. 792, Corigliano d'Otranto - P. 748) e di un'isola linguistica albanese (Acquaformosa - P. 751). ${ }^{13}$ L'inserzione dei rispettivi potenziali linguistici nella rete dell'AIS offre molti spunti per una miglior conoscenza del contatto linguistico instauratosi tra le parlate delle rispettive isole linguistiche e la loro vicinanza dialettale autoctona.

Quanto ai tre punti galloitalici dell'AIS ed all'affidabilità della rispettiva documentazione nell'AIS, Gerhard Rohlfs, l'autore delle tre inchieste, si esprime nel manuale introduttivo dell'AIS nei seguenti termini:

Aidone (P. 865):

«Il dialetto gallo-italico è in netto regresso perché il siciliano viene usato anche dai contadini nei rapporti con i 'signori'. Ne consegue che ci sono spesso più modi per indicare le stesse cose.» (Jaberg/Jud 1987 [ital.]: 165; cf. anche Jaberg/Jud 1928 [ted.]: 134)

11 Ovviamente la stessa carta originale dell'AIS (27 il suo cognato) può essere utilizzata anche per altre analisi tassatorie di stampo fonetico, p. es. per l'accertamento degli esiti dialettali dei nessi latini -O-, -GN-, -A- o -T-, tutti compresi nell'etimo latino COGNÁTU.

$12 \mathrm{Si}$ tratta di un'isola linguistica di origine pittavina (Andraut, P.-ALF 635) che fu fondata nel XV secolo e si trova in territorio guascone. Per un'analisi DM della sua posizione in seno alla rete dell'ALF cf. Goebl (2002: 23, 54; carta 13).

13 Per un'analisi DM delle posizioni relazionali di Guardia Piemontese (P.-AIS 760), Ghorio (P.AIS 792) e Acquaformosa (P.-AIS 751) cf. Goebl (2008: 61-64). 
Sperlinga (P. 836):

«L'antico dialetto gallo-italico viene ancora parlato da tutti gli strati della popolazione; anche se il siciliano è ancora poco usato, il dialetto locale, per l'influsso del siciliano e dell'italiano, va incontro a un lento decadimento.» (Jaberg/Jud 1987 [ital.]: 163; cf. anche Jaberg/Jud 1928 [ted.]: 133)

San Fratello (P. 817):

«Il dialetto gallo-italico parlato da tutti gli abitanti del luogo è molto nettamente distinto dal siciliano, che penetra nel ceto borghese della città e viene anche usato nei rapporti con persone di altre località.» (Jaberg/Jud 1987 [ital.]: 161; cf. anche Jaberg/Jud 1928 [ted.]: 131)

Il fatto della più o meno grande ibridizzazione dei dialetti galloitalici in Sicilia e nell'Italia meridionale è riconosciuto da tutti gli autori competenti. Ciononostante la ricostruzione il più precisa possibile della loro madrepatria era sempre lo scopo maggiore (per non dire «nobile») delle molteplici analisi linguistiche effettuate in merito. Visto che dal periodo della colonizzazione galloitalica in Sicilia e nell'Italia meridionale, avvenuta nel XII e XIII secolo, sono trascorsi otto o nove secoli, e considerato che nel frattempo anche la configurazione dialettale della patria dei coloni galloitalici non è rimasta inalterata, il tentativo di voler evidenziare con metodi meramente linguistici i nuclei di provenienza dei coloni galloitalici nel tessuto dei dialetti dell'Italia settentrionale odierna non può che risultare altamente problematico. Ciò non impedisce che molte delle risposte date finora con mezzi qualitativi, siano abbastanza convincenti e combacino pure, benchè in varia misura, con i risultati di analoghe ricerche storiche.

In questa sede diamo per scontata l'opinio communis in merito depositata, tra l'altro, negli scritti di Giulia Petracco Siccardi (1965), Max Pfister (1988), Gerhard Rohlfs (1965 e 1975) e di Salvatore C. Trovato (1989, 1998 e 1999). Vediamo ora in che maniera la DM possa risultare utile in merito tramite l'applicazione di quattro dei suoi metodi analitici.

\section{SGUARDO DIALETTOMETRICO A: IL MESSAGGIO GEOLINGUISTICO DELLE CARTE DI SIMILARITÀ}

La carta di similarità costituisce - metaforicamente parlando - la «colonna vertebrale» dell'euristica intera della SDMS. Ciascuna carta di similarità dispone di un punto di riferimento preselezionato e visualizza, tramite lo spiegamento spaziale della sua colorazione graduata, il calo progressivo dei valori della similarità linguistica rispetto al valore supremo di $100 \%$ del punto di riferimento in questione. Accenniamo, per una miglior comprensione delle carte di similarità, a due fatti cartografici di rilievo:

- Il poligono del punto di riferimento rimane sempre in bianco. 
- Il poligono bianco del punto di riferimento si trova in genere nel centro o al margine della zona rossa che corrisponde ai punti-AIS provvisti dei più alti tassi di similarità.

Grazie ai loro profili coropletici molto netti, le carte di similarità dimostrano, in chiave geolinguistica, le seguenti proprietà del rispettivo dialetto (basiletto) locale:

- la sua posizione relazionale all'interno della rete esaminata,

- il tasso di diffusione (penetrazione, interazione, ecc.) della sua dialettalità all'interno della rete esaminata,

- la potenza comunicativa del rispettivo dialetto.

I calcoli di similarità sono stati effettuati mediante l'indice di similarità standard della SDMS IRI $I_{k}$ («Indice Relativo d'Identità» tra le località $j$ e $k$ ). Anche la visualizzazione dei valori calcolati segue le norme abituali della SDMS: vengono applicati gli algoritmi MINMAMAX e MEDMA,${ }^{14}$ sempre sulla base di sei gradini cromatici (e/o numerici), il che agevola enormemente la percezione visiva univoca del profilo coropletico da parte dell'osservatore.

Siccome è spesso necessario poter comparare i profili coropletici tra di loro, abbiamo allestito la loro presentazione in maniera sinottica: in questo modo è possibile comparare sempre tra di loro quattro profili DM.

\subsection{La posizione geolinguistica relazionale di Aidone (e dintorni): dati integrali (3911 CL)}

Si vedano le carte 3 e 4 .

Si tratta di due analisi di similarità basate su tutti i dati disponibili (=3911 CL). La carta 4 mostra un profilo coropletico tipicamente «siciliano». I colori caldi (che corrispondono sempre ai valori situati sopra la media aritmetica della rispettiva distribuzione di similarità) si stendono fino (e non oltre) la «linea La Spezia - Rimini»; i rispettivi tassi di similarità diminuiscono lentamente da sud verso nord. La carta 3 esibisce invece un profilo tipicamente «galloitalico» contrassegnato soprattutto dall'inclusione di vaste aree dell'Italia settentrionale nella zona gialla, e dalla presenza di valori di similarità eccezionalmente alti nella Padania. I tassi di similarità più alti si trovano, su ambedue le carte, nella immediata vicinanza del punto di riferimento: sulla carta 3 si tratta del punto 836 , Sperlinga, cioè di un altro punto-AIS di stampo galloitalico; sulla carta 4 la stessa funzione viene svolta da un altro punto-AIS di tipo siciliano (851 - San Biagio Platani). Sulla carta 3, i poligoni gialli situati nel Piemonte poggiano su valori di similarità solo leggermente superiori a quelli rilevati nel Veneto.

\footnotetext{
14 Nella versione tedesca di VDM i rispettivi algoritmi vengono chiamati MINMWMAX e MEDMW. Si noti che le sigle MW e MA equivalgono alle parole «Mittelwert» (ted.) e «media aritmetica» (ital.).
} 
Un'analisi più accurata dei tassi di similarità relativi alle zone in rosso e arancione situate in Sicilia e nel sud d'Italia da una parte ed alle zone gialle della Padania dall'altra, permette di accertare, tramite il numero delle CL coinvolte nelle rispettive misurazioni della similarità, la quota della meridionalizzazione del dialetto di Aidone da un lato e quella della conservazione di tratti linguistici di stampo padano dall'altro. Mentre nel vettore-attributi del dialetto di Aidone il numero delle CL di tipo meridionale ammonta a ca. 400 (su 3911) unità, il numero delle CL di tipo padano (cioè conservatore) comprende solo 60 (su 3911) unità. La relazione numerica tra innovazione e tradizione/ritenzione s'aggira dunque su $6: 1$.

Questo bilancio quantitativo non si presta all'accertamento puntuale della patria linguistica dei primi abitanti di Aidone.

\subsection{La posizione geolinguistica relazionale di Sperlinga (e dintorni): dati integrali (3911 CL)}

Si vedano le carte 5 e 6 .

Mentre per la vicinanza dialettale siciliana di Sperlinga il rispettivo profilo coropletico (si veda la carta 6) è molto simile a quello della carta 4 , si evidenzia una configurazione alquanto diversa del profilo coropletico della carta 5 dove la presenza dei poligoni gialli è stata ristretta alla Liguria ed al Veneto. Quanto ai rispettivi tassi di similarità, i valori relativi alla Liguria oltrepassano solo leggermente quelli relativi al Veneto.

Una volta di più questo stato delle cose non fornisce ulteriori chiarimenti sulla provenienza esatta dei primi coloni di Sperlinga.

\subsection{La posizione geolinguistica relazionale di San Fratello (e dintorni): dati integrali (3911 CL)}

Si vedano le carte 7 e 8 .

Le strutture coropletiche delle carte 7 e 8 assomigliano molto a quelle delle carte 3 e 4. Di nuovo possiamo constatare un radicamento oltremodo solido della dialettalità di San Fratello in alcune zone della Padania ed anche la presenza delle consuete caratteristiche di una carta di similarità: 1) decremento dei tassi di similarità nello spazio, 2) strutturazione coropletica molto netta della carta intera.

Alla pari delle carte 3 e 5, anche la carta 7 non si presta all'individuazione precisa della madrepatria linguistica dei sanfratellini.

Un'ulteriore modalità di affrontare il problema dell'inserzione della dialettalità di una singola isola linguistica nella rete dell'AIS consiste nell'esaminare separatamente il rispettivo comportamento DM delle diverse categorie linguistiche, in ispecie della fonetica e del lessico.

\subsection{La posizione geolinguistica relazionale di Aidone: dati fonetici vs. lessicali}

Si vedano le carte 9 e 10 .

Abbiamo utilizzato per la messa a punto della carta 9 il nostro corpus fonetico con $1766 \mathrm{CL}$, e per quella della carta 10 il corpus lessicale con $1225 \mathrm{CL}$. Le dimensioni quantitative di ambedue i corpora sono tali da rendere molto affidabile il paragone 
visivo dei due profili coropletici. Dal loro confronto risulta chiaramente che la parlata di Aidone ha tuttora conservato il suo carattere «nordico» dal punto di vista fonetico, ma che tale non è il caso per il lessico, contrassegnato da un adeguamento linguistico pressochè totale alla vicinanza di tipo siciliano. I profili coropletici di stampo lessicale fatti a partire dai punti di riferimento siciliani attigui a Aidone, dimostrano strutturazioni praticamente identiche a quella della carta 10.

I valori-IRI più alti della Padania si ritrovano nel Piemonte, in Lombardia ed anche nel Trentino. Una riconduzione diretta della parlata di Aidone ad una zona chiaramente circoscritta della Padania non è pertanto possibile.

\subsection{La posizione geolinguistica relazionale di Sperlinga: dati fonetici vs. lessicali}

Si vedano le carte 11 e 12 .

Il contrasto tra i profili coropletici delle carte 11 e 12 è sostanzialmente lo stesso di prima. La configurazione del profilo coropletico della carta 11, dove si evidenzia una concentrazione insolita di poligoni in arancione tanto nell'Italia mediana quanto nel Veneto, ${ }^{15}$ ci induce ad avanzare l'ipotesi di un certo sbandamento italianeggiante dell'inchiesta condotta da G. Rohlfs a Sperlinga nell'aprile del 1924. Un'altra spia del carattere «troppo italiano» dell'inchiesta fatta a Sperlinga è la scarsa similarità fonetica rilevabile tra Sperlinga e l'isola linguistica provenzale di Guardia Piemontese (P.AIS 760), il cui poligono (reperibile sulla carta 11 immediatamente sotto la menzione «Basilicata») è contrassegnato solo in verde e dispone perciò di un tasso di similarità situato sotto la rispettiva media aritmetica.

\subsection{La posizione geolinguistica relazionale di San Fratello: dati fonetici vs. lessicali}

Si vedano le carte 13 et 14 .

I risultati della doppia analisi di similarità (relativa cioè alla fonetica ed al lessico) si allineano perfettamente a quelli relativi alla situazione di Aidone. Si evidenzia di nuovo la ormai ben nota propensione delle isole linguistiche a conservare in genere molto meglio le caratteristiche fonetiche (ed anche morfologiche) della rispettiva parlata di origine che non quelle lessicali.

Anche a San Fratello la nostra previa esperienza di una localizzazione precisa della madrepatria linguistica di un'isola linguistica, fatta sulla base dei dati di Guardia Piemontese (cf. Goebl 2008: 61-62), non può essere reiterata.

\section{SGUARDO DIALETTOMETRICO B: IL MESSAGGIO GEOLINGUISTICO DELLE CARTE INTERPUNTUALI (CARTA ISOGLOTTICA E CARTA A RAGGI)}

Si vedano le carte 15 e 16 .

Dal punto di vista cartografico le carte interpuntuali si differenziano molto dalle carte coropletiche. Rientrano nella classe delle carte «isaritmiche», i cui elementi

15 Per la posizione geotipologica «mediana» dei dialetti veneti cf. Goebl (2008: 58-61, 104-107; con le tavole 29-36). 
iconici di base sono o i lati di triangolo o i lati di poligono generati ambedue tramite un previo trattamento geometrico della rete esaminata. La confezione delle carte 15 e 16 si basa quindi sulle tappe seguenti: 16

- triangolazione della rete-AIS secondo i principi della geometria di Delaunay (cf. Okabe/Boots/Sugihara 1992: 72 ss.): ne risultano - per la rete 1 e la carta 16 970 lati di triangolo.

- poligonizzazione successiva della rete triangolata secondo i principi della geometria di Voronoi (cf. Okabe/Boots/Sugihara 1992: 65 ss.): ne risultano - per la rete 1 e la carta 15 - 970 lati di poligono.

- allestimento dei mezzi cartografici atti a conferire parallelamente ai lati di triangolo o di poligono uno spessore ed una colorazione variabile a seconda della grandezza (o meno) dei rispettivi valori interpuntuali da visualizzare. Il programma VDM consente la realizzazione rapida di ambedue le possibilità.

Dal punto di vista tasso- o dialettometrico la carta 15 è basata su 970 valori non di similarità bensì di distanza. È stato applicato in merito l'«Indice Relativo di Distanza» $\left(\operatorname{IRD}_{\mathrm{jk}}\right.$, i cui valori oscillano tra 10,19 e 47,52\%. L'IRD è il complemento dell'IRI secondo la formula seguente: IRD + IRI $=100$. La visualizzazione dei suddetti 970 valori viene effettuata lungo altrettanti lati di poligoni secondo il principio cartografico seguente: più i valori dell'IRD sono alti, più i rispettivi lati di poligono sono spessi e si accostano al colore blu (scuro). E viceversa. In questa maniera si manifestano, in non poche zone della nostra carta, fenomeni con apparenze lineiformi equivalenti ai ben noti «fasci d'isoglosse». ${ }^{17}$ Questo si verifica al sud della Rumantschia, lungo l'arco alpino occidentale, all'interno della Padania (intorno alla Lombardia ed al Veneto), lungo l'Appennino, nella Sardegna settentrionale ed intorno a tutte le isole linguistiche del sud e della Sicilia. Mentre la configurazione lineiforme spessa e contrassegnata in blu scuro situata lungo l'Appennino tosco-emiliano evoca la ben nota «linea La SpeziaRimini» di memoria wartburghiana, ${ }^{18}$ la sua contropartita insediata tra Roma ed Ancona offre un assetto tutt'altro che lineare. Si tratta piuttosto di una larga zona di passaggio con svariati effetti di compartimentalizzazione a breve distanza.

Le zone dove si accumulano i poligoni sottili contrassegnati in rosso rimandano al contrario ai nuclei dialettali ben visibili sulla carta 16 .

Dal contrasto delle carte 15 e 16 emerge la differenza fondamentale che intercorre tra le carte isoglottiche e le carte a raggi. Mentre sulla carta 15 si evidenziano feno-

16 Per il problema delle carte interpuntuali cf. soprattutto Goebl (1983: passim), nonché (1984a I: 183-196).

17 Rinviamo, per motivi storici, alla sinossi isoglottica, basata sui dati dell'AIS e relativa all'Italia del nord, che è stata pubblicata nel 1930 dal germanista Karl Haag ed al fatto che lo stesso autore ha utilizzato per primo - ovviamente avant la lettre - i principi della geometria di DelaunayVoronoi per la poligonizzazione di una rete atlantistica (cf. Haag 1898).

18 Per la storia dei concetti delle «linee» La Spezia-Rimini e Roma-Ancona cf. Goebl (2003b: 634). 
meni di confinazione e di distanziazione interpuntuale, dalla carta 16 si ricavano fatti inversi: collegamenti linguistici a brevissima distanza. Tramite l'addensamento spaziale spiccato di molti triangoli spessi e contrassegnati in rosso il profilo isaritmico della carta 16 mostra l'ubicazione di nuclei dialettali. Per una miglior comprensione della carta 16 può giovare anche l'idea metaforica che ciascuno dei 970 triangoli funge da «tubo» posto tra due località contigue nel quale corrono «flussi» di similarità linguistica di varia intensità.

Bisogna però aggiungere che, dal punto di vista tassometrico, l'analisi interpuntuale è piuttosto superficiale. In effetti, i 970 valori interpuntuali rappresentano, sui 72771 valori delle rispettive matrici di distanza o di similarità, ${ }^{19}$ solo 1'1,33\% della totalità delle informazioni quantitative disponibili.

\section{SGUARDO DIALETTOMETRICO C: IL MESSAGGIO GEOLINGUISTICO DI UNA CARTA A PARAMETRI (SINOSSI DEI COEFFICIENTI DI ASIMMETRIA DI FISHER [CAF] DELLE 382 DISTRIBUZIONI DI SIMILARITÀ)}

Si veda la carta 17.

La natura statistica molto variabile delle $\mathrm{N}$ distribuzioni di similarità delle quali consiste ciascuna matrice di similarità (con le dimensioni $\mathrm{N} \times \mathrm{N}$ ), ci ha ben presto indotto ad esaminare più da vicino - sempre in chiave comparativa - i valori dei diversi parametri statistici (come il minimo, il massimo, la media aritmetica, ecc.). ${ }^{20}$ $\mathrm{Nel}$ corso di queste ricerche si è rivelata la grande utilità geolinguistica di alcuni parametri relativi alla simmetria della rispettiva distribuzione di similarità. La loro visualizzazione sinottica permette di evidenziare il fenomeno del «compromesso linguistico» (ted. «Sprachausgleich ${ }^{21 »)}$.

Lo studio dialettometrico dello «Sprachausgleich» («compromesso linguistico») costituisce uno dei capitoli più affascinanti della DM salisburghese, tanto dal punto di vista meramente geolinguistico quanto da quello della tassometria propriamente detta. Per «Sprachausgleich» - che ovviamente dispone di diversi gradi di intensità - intendiamo la più o meno forte integrazione connettiva di un particolare basiletto nella totalità della rispettiva rete geolinguistica. Pare logico - e corrisponde inoltre alla nostra esperienza empirica - che tra i diversi basiletti di una rete geolinguistica data ce ne siano alcuni che assumono un rilievo molto isolato rispetto ai loro omologhi ed altri i cui legami con la maggioranza delle componenti della corrispondente rete sono molto intensi.

Ecco una riflessione per facilitare la comprensione di questo fenomeno. Ciascuno dei 382 vettori di altrettante località della rete 1 del progetto-DM qui presentato, è contrassegnato da 3911 attributi (o tassati) geolinguistici. Orbene, ciascu-

\footnotetext{
19 Il valore di 72771 si calcola tramite la seguente formula combinatoria: $\mathrm{N} / 2 \times(\mathrm{N}-1)$, per $\mathrm{N}=382$.

20 Per ulteriori dettagli cf. Goebl (1984a I: 136-164).

${ }^{21} \mathrm{Si}$ tratta di un concetto (geo)linguistico coniato in seno agli studi germanici: cf. Besch (1967: passim).
} 
no di questi 3911 tassati corrisponde ad un'area geolinguistica la cui estensione può variare - teoricamente - tra 1 e 381 punti (o poligoni). ${ }^{22}$ È quindi di somma importanza, per il livello d'integrazione di uno dei $\mathrm{N}$ vettori nella rispettiva rete, la ripartizione percentuale di aree con estensioni minori, medie o maggiori. Da questa considerazione si evidenzia che un punto-vettore che dispone prevalentemente di aree con un'estensione molto ridotta non può che giocare un ruolo comunicativo molto limitato in seno alla rete intera. E viceversa.

Gli strumenti statistici più adatti alla rilevazione di questo fenomeno in sede di statistica sono gli indici per la misurazione della più o meno grande simmetria delle rispettive distribuzioni di similarità. Nei termini della statistica descrittiva una distribuzione di frequenza viene considerata come «simmetrica» quando la metà dei valori numerici che la costituiscono si trovano sotto e l'altra metà dei valori sopra la rispettiva media aritmetica. Tra le diverse possibilità offerte dalla statistica per la misurazione della simmetria spicca il «coefficiente di asimmetria di Fisher» (CAF: ingl. «skewness», ted. «Schiefe»; cf. Brentari 1990: 40-41.).

Il coefficiente di asimmetria di Fisher $(\mathrm{CAF})^{23}$ assume il valore 0 per una simmetria totale della rispettiva distribuzione di frequenza, valori negativi nel caso di una dislocazione (asimmetrica) verso destra (maggioranza dei valori sopra la media aritmetica) e valori positivi nel caso di una dislocazione (asimmetrica) verso sinistra (maggioranza dei valori sotto la media aritmetica).

Sulla carta 17, ai valori negativi corrispondono i poligoni in blu scuro e medio, mentre i valori positivi vengono marcati dai poligoni in rosso ed arancione.

Ecco il significato linguistico dei colori:

- poligoni in blu (ecc.):

zone con un tasso molto alto del compromesso linguistico (Sprachausgleich), contrassegnate in altre parole - tanto in dia- quanto in sincronia - da un gran dinamismo interattivo rispetto alla totalità della rete dell'AIS. ${ }^{24} \mathrm{Si}$ tratta dunque di zone molto attive tanto dal punto di vista espansivo quanto ricettivo.

- poligoni in rosso (ecc.):

zone con un tasso molto basso del compromesso linguistico (Sprachausgleich), caratterizzate quindi - tanto in dia- quanto in sincronia - da un grande conservatorismo linguistico rispetto alla totalità della rete esaminata. Si tratta pertanto di zone la cui tipicità è stata preservata per molto tempo ed in larga misura da intrusioni linguistiche di qualsiasi origine.

Uno sguardo alla carta 17 ci rivela che la ripartizione spaziale dei colori blu e rosso (ed anche delle tinte intermedie) è molto chiara. Nella Padania, i poligoni in blu scuro e medio creano una dipartimentalizzazione ben definita dove emergono, come

22 L'estensione massima di $\mathrm{N}$, cioè di 382 punti, non può essere ammessa in questa sede perché significherebbe un azzeramento totale della variabilità geolinguistica.

23 Per la formula del CAF cf. Goebl (1981: 394) e (1984a I: 150).

24 Il rispettivo dinamismo interattivo non si limita dunque alla vicinanza immediata dei punti della rete, bensì abbraccia sempre la totalità di tutti i punti d'inchiesta della rete. 
zone divisorie provviste però di altissimi tassi interattivi, il corso dell'Adige e del Po nonché lunghi tratti della catena appenninica dalla Liguria fino alle Marche. Un altro solco vallivo blu (e quindi provvisto di un alto potenziale interattivo) s'incunea tra l'Italia mediana (Lazio, Umbria, Marche) e quella del sud (Abruzzo e Molise, Campania, ecc.). Le zone in rosso (ecc.) rappresentano invece configurazioni non lineiformi bensì spiccatamente puntuali, in altre parole provviste di un'estensione corica geograficamente coerente.

È ovvio che le isole linguistiche romanze situate nel sud (PP. 715 - Faeto, e 760 Guardia Piemontese) ed in Sicilia (PP. 865, 836 e 817) e soggette sin dalla loro genesi a perpetui interscambi linguistici sono contrassegnate in blu. Lo stesso dicasi per la parte settentrionale della Sardegna, la quale, considerando la sua (rampante) toscanizzazione plurisecolare tramite la Corsica (cf. Wagner 1959: 392-396), dispone, in ultima analisi, di molte caratteristiche di un'isola linguistica vera e propria.

\section{SGUARDO DIALETTOMETRICO D: IL MESSAGGIO GEOLINGUISTICO DI UNA CARTA A CORRELAZIONI}

La DM correlativa è stata aggiunta solo nel 2004 all'armamentario metodico della SDMS. ${ }^{25}$ È nata dallo studio approfondito delle relazioni che, senza ombra di dubbio, intercorrono tra la distanza (o prossimità) geografica e quella linguistica. Questo si evidenzia subito da una considerazione sinottica di molte carte di similarità. Per ottenere una visione più precisa delle relazioni che esistono tra la similarità linguistica e quella geografica è possibile affiancare allo strumento euristico della carta di similarità quello della carta di prossimità. Mentre la carta di similarità rappresenta - sempre a partire da un punto di riferimento preselezionato - il calo (spesso disuguale) della similarità linguistica nello spazio geografico, la carta di prossimità visualizza il calo (assolutamente regolare) delle prossimità chilometriche rispetto al punto di riferimento preselezionato. Il calcolo delle rispettive prossimità viene eseguito secondo il noto teorema di Pitagora e quindi secondo i principi della geometria euclidea. Siccome le coordinate geografiche $(x$ e $y)$ dei punti dell'AIS sono già a disposizione del programma VDM, la successiva applicazione del teorema di Pitagora risulta molto facile.

Per l'accertamento delle (cor)relazioni esistenti tra le dimensioni della «lingua» e dello «spazio» sarebbe possibile, almeno in teoria, esaminare de visu, nel quadro di un qualsiasi progetto-DM, $\mathrm{N}$ coppie di carte di similarità e di prossimità. Ovviamente questa considerazione meramente visuale e quindi impressionistica, non condurrebbe a risultati scientificamente «probanti». Dal momento che la statistica moderna offre la possibilità di misurare la relazione tra due serie di valori empiricamente rilevati mediante appositi indici di correlazione, l'applicazione di tali misure e la successiva visualizzazione sinottica dei rispettivi risultati potrebbero costituire una soluzione di gran lunga preferibile alla suddetta comparazione visiva

25 Per una prima presentazione delle molteplici possibilità della DM correlativa rinvio alla nostra grande relazione di lavoro del 2005, redatta in francese, ed anche alla rispettiva presentazione italiana del 2007. 
reiterata di profili coropletici accoppiati. Il calcolo delle correlazioni si fa a partire da due matrici quadrate dalle dimensioni $\mathrm{N} \times \mathrm{N}$ (relative cioè alla similarità linguisti$c a$ da una parte ed alla prossimità euclidea dall'altra) e fornisce come risultato $\mathrm{N}$ valori di correlazione che vengono quindi cartografati nella forma abituale. ${ }^{26}$

La carta 18 rivela una ripartizione spaziale molto netta di poligoni contrassegnati in blu (ecc.) ed in rosso (ecc.). La significazione linguistica dei rispettivi colori è questa:

- colori caldi (rosso, ecc.):

zone dove persiste un'armonia originaria tra «lingua» e «spazio» e dove si sono effettuati pochi trasferimenti di fonemi o lessemi in contrasto con lo scambio linguistico di stampo «naturale». Si tratta cioè di zone conservatrici, rimaste linguisticamente quiete e piuttosto incontaminate.

- colori freddi (blu, ecc.):

zone dove un'eventuale armonia originaria tra «lingua» e «spazio» è stata profondamente perturbata «da fuori», tramite l'ingerenza di dinamismi culturali, sociali o politici di varia origine. Si tratta di zone linguisticamente inquiete, pervase da dinamismi (socio)linguistici anche di lunga durata.

Un rapido sguardo alla carta 18 pone in evidenza che le zone »inquiete» si collocano soprattutto nella parte settentrionale della Toscana, in Liguria e nel Veneto (con inclusione dell'Istria) e nella Sardegna settentrionale. Dalla stessa carta si ricava inoltre il fatto - d'altronde molto plausibile - che le cinque isole linguistiche nel sud ed in Sicilia costituiscono - una volta di più - zone linguisticamente «movimentate». Ricordiamo in merito che la fondazione di un'isola linguistica rappresenta, in effetti, una rottura totale di un'eventuale armonia originaria tra lingua e spazio.

\section{CONCLUSIONE}

Arrivati al termine di questa presentazione sommaria di alcuni capi della dialettometria salisburghese, ci preme di riaffermare alcune caratteristiche salienti della suddetta:

- si tratta di un metodo induttivo che, tramite la sintesi quantitativa di dati empirici molto variegati, porta alla scoperta di tendenze, anzi leggi geolinguistiche, celate a prima vista nei dati analizzati;

- si tratta di un metodo orientato verso l'analisi statistica dello spazio e che, per questo motivo, ha fatto della cartografazione (o visualizzazione) generale dei suoi risultati numerici la via regia della propria euristica;

- si tratta di un metodo che, grazie alla sua impostazione comparativa intrinseca, rimane sempre aperto a cooperazioni interdisciplinari di qualsiasi natura;

- si tratta di un metodo la cui base empirica è costituita dagli atlanti linguistici propriamente detti ed anche da analoghe collezioni empiriche;

26 Si vedano i rispettivi schemi grafici esplicativi in Goebl (2005: 328) e (2007: 47). 
- si tratta di un metodo che, nato in seno alla Romanistica, rientra nella tradizione della geografia linguistica classica e si vanta di conciliare armonicamente saperi tradizionali ed innovazioni metodiche, nonché tecnologiche, di recente data.

- Quanto alle tre isole linguistiche galloitaliche comprese nella rete dell'AIS, le quattro analisi DM applicate consentono sì di evidenziare il loro carattere «nordico», non però di fornire ulteriori elementi per la loro localizzazione precisa nel tessuto dialettale della Padania.

\section{Ringraziamenti}

- Sussidio finanziario:

Finanziamento del progetto di ricerca no. 18365 da parte del «Fonds zur Förderung der wissenschaftlichen Forschung in Österreich»(FWF), Vienna [Fondazione austriaca per la ricerca scientifica]

- Tassazione delle 3911 carte di lavoro (CL):

I lavori di tassazione sono stati eseguiti dai seguenti romanisti salisburghesi: Emese Lörincz, Christine Greil, Julia Hattinger, Daniel Rötzer, Mirjam Högl e Gertraud Klingler. La correzione dei dati tassati è stata resa possibile tramite un programma informatico creato da Xavier Casassas.

- Ideazione, creazione e aggiornamento continuo del programma VDM (Visual DialectoMetry):

Edgar Haimerl, Seattle (Stati Uniti d'America)

- Mantenimento e cura del programma VDM (Visual DialectoMetry) a Salisburgo: Slawomir Sobota, Salisburgo

- Realizzazione delle 18 carte a colori di questo contributo:

Werner Goebl, Vienna

- Revisione stilistica del nostro testo italiano:

Adelaide Fiocchi Baehr, Salisburgo.

A tutte le persone citate, nonché all'ente di ricerca FWF vada l'espressione della mia sincera e profonda gratitudine.

\section{Bibliografia}

AIS $=$ JABERG, Karl/Jakob Jud (a cura di) (1928-1940) Sprach- und Sachatlas Italiens und der

Südschweiz. 8 voll. Zofingen: Ringier. [Ristampa: Nendeln /Liechtenstein/, Kraus, 1971]. Alf = Gilliéron, Jules/Edmond Edmont (a cura di) (1902-1910) Atlas linguistique de la France. 10 voll. Paris: Champion. [Ristampa: Bologna, Forni, 1968].

AsColI, Graziadio Isaia (1876) «Del posto che spetta al ligure nel sistema dei dialetti italiani.» Archivio glottologico italiano 2, 111-140.

BAUER, Roland (2003) «Sguardo dialettometrico su alcune zone di transizione dell'Italia nordorientale (lombardo vs. trentino vs. veneto).» In: R. Bombi/F. Fusco (a cura di), Parallela $X$. Sguardi reciproci. Vicende linguistiche e culturali dell'area italofona e germanofona. Udine: Forum, 93-119. 
BAUER, Roland (2005) «La classificazione dialettometrica dei basiletti altoitaliani e ladini rappresentati nell'Atlante linguistico del ladino dolomitico e dei dialetti limitrofi (ALD-I).» In: C. Guardiano et al. (a cura di), Lingue, istituzioni, territori. Riflessioni teoriche, proposte metodologiche ed esperienze di politica linguistica. Roma: Bulzoni, 347-365.

BAUER, Roland (2009) Dialektometrische Einsichten. Sprachklassifikatorische Oberflächenmuster und Tiefenstrukturen im lombardo-venedischen Dialektraum und in der Rätoromania. S. Martin de Tor: Istitut Ladin «Micurà de Rü». (Ladinia monografica, 01).

BESCH, Werner (1967) Sprachlandschaften und Sprachausgleich im 15. Jahrhundert. Studien zur Erforschung der spätmittelalterlichen Schreibdialekte und zur Entstehung der neuhochdeutschen Schriftsprache. München: Francke.

Bock, Hans Hermann (1974) Automatische Klassifikation. Theoretische und praktische Methoden zur Gruppierung und Strukturierung von Daten (Cluster-Analyse). Göttingen: Vandenhoeck \& Ruprecht.

Brentari, Eugenio (1990) Asimmetria e misure di asimmetria. Torino: Giappichelli.

Brun-Trigaud, Guylaine/Yves Le Berre/Jean Le Dû (2005) Lectures de l'Atlas linguistique de la France de Gilliéron et Edmont. Du temps dans l'espace. Essai d'interprétation des cartes de l'Atlas linguistique de la France de Jules Gilliéron et Edmond Edmont augmenté de quelques cartes de l'Atlas linguistique de la Basse-Bretagne de Pierre Le Roux. Paris : Editions du CTHS.

Chandon, Jean-Louis/Suzanne Pinson (1981) Analyse typologique. Théories et applications. Paris/New York/Barcelona/Milano: Masson.

Goebl, Hans (1981) «Éléments d'analyse dialectométrique (avec application à 1'AIS).» Revue de linguistique romane 45, 349-420.

Goebl, Hans (1982) Dialektometrie. Prinzipien und Methoden des Einsatzes der Numerischen Taxonomie im Bereich der Dialektgeographie. Wien: Österreichische Akademie der Wissenschaften, 1-123. [41 tavole. Denkschriften, philosophisch-historische Klasse; vol. 157].

Goebl, Hans (1983) «Parquet polygonal et treillis triangulaire : les deux versants de la dialectométrie interponctuelle.» Revue de linguistique romane 47, 353-412.

Goebl, Hans (1984a) Dialektometrische Studien. Anhand italoromanischer, rätoromanischer und galloromanischer Sprachmaterialien aus AIS und ALF. 3 voll. Tübingen: Niemeyer.

GoEBL, Hans (1984b) «Lineamenti di dialettometria (con applicazione all'AIS).» Guida ai dialetti veneti $\mathrm{VI}, 7-53$.

Goebl, Hans (1986) «Considérations dialectométriques sur le problème de 1"unité rhétoromane (ladine)'.» Linguistica 26, 83-97.

Goebl, Hans (1987) «Points chauds de l'analyse dialectométrique: pondération et visualisation.» Revue de linguistique romane 51, 63-118.

Goebl, Hans (1988) «Il posto dialettometrico che spetta ai punti-AIS 338 (Adorgnano, Friuli), 398 (Dignano/Vodnjan, Istria) e 367 (Grado, Friuli). Presentazione di tre carte di similarità.» Linguistia 28, 75-103.

Goebl, Hans (1991) «Una classificazione gerarchica di dati geolinguistici tratti dall'AIS. Saggio di dialettometria dendrografica.» Linguistica 31, 341-351.

Goebl, Hans (2000) «La dialectométrisation de l'ALF: présentation des premiers résultats.» Linguistica 40, 209-236. 
Goebl, Hans (2002) «Analyse dialectométrique des structures de profondeur de l'ALF.» Revue de linguistique romane 66, 5-63.

Goebl, Hans (2003a) «Regards dialectométriques sur les données de l'Atlas linguistique de la France (ALF): relations quantitatives et structures de profondeur.» Estudis Romànics 25, 59-120.

Goebl, Hans (2003b) «Entwicklung der romanischen Sprachgrenzen: Italoromania und Ostalpenraum.» In: G. Ernst et al. (a cura di), Romanische Sprachgeschichte. Histoire linguistique de la Romania. Ein internationales Handbuch zur Geschichte der romanischen Sprachen. Manuel international d'histoire linguistique de la Romania. Vol. I. Berlin/New York: de Gruyter, 631-645.

Goebl, Hans (2004) «VDM - Visual DialectoMetry. Vorstellung eines dialektometrischen Software-Pakets auf CD-ROM (mit Beispielen zu ALF und Dees 1980).» In: W. Dahmen et al. (a cura di), Romanistik und neue Medien: Romanistisches Kolloquium XVI. Tübingen: Narr, 209-241.

Goebl, Hans (2005) «La dialectométrie corrélative: un nouvel outil pour l'étude de l'aménagement dialectal de l'espace par l'homme.» Revue de linguistique romane 69, 321-367.

Goebl, Hans (2006a) «Recent Advances in Salzburg Dialectometry.» Literary and Linguistic Computing 21/4, 411-435.

GoebL, Hans (2006b) «Sur le changement macrolinguistique survenu entre 1300 et 1900 dans le domaine d'Oïl. Une étude diachronique d'inspiration dialectométrique.» Linguistica 46, 3-43.

Goebl, Hans (2007a) «Sprachgeographische Streifzüge durch das Netz des Sprachatlasses AIS.» Ladinia XXXI, 187-271.

Goebl, Hans (2007b) «Breve presentazione della dialettometria correlativa.» In: V. Matranga/R. Sottile (a cura di), Esperienze linguistiche. Percorsi di ricerca italiani e europei. Atti del Seminario di Studi su Percorsi di Geografia linguistica. Esperienze italiane e europee. (Palermo, 23-24 marzo 2005). Palermo: Centro di Studi Filologici e Linguistici Siciliani, 43-61.

Goebl, Hans (2008) «La dialettometrizzazione integrale dell'AIS. Presentazione dei primi risultati.» Revue de Linguistique Romane 72, 25-113.

HAAG, Carl (1898) Die Mundarten des oberen Neckar- und Donautales. (Schwäbisch-alemannisches Grenzgebiet: Baarmundarten). Reutlingen: Hutzler.

HAAG, Karl (1930) «Die Sprachlandschaften Oberitaliens (mit einer Karte).» Germanischromanische Monatsschrift 18, 458-478.

HeERINGA, Wilbert (2004) Measuring Dialect Pronunciation Differences using Levenshtein Distance. Groningen: University of Groningen. (Groningen Dissertations in Linguistics, 46).

JABERG, Karl (1936) Aspects géographiques du langage. Paris: Droz.

JABERG, Karl/Jakob Jud (1928) Der Sprachatlas als Forschungsinstrument. Kritische Grundlegung und Einführung in den Sprach- und Sachatlas Italiens und der Südschweiz. Halle: Niemeyer. [Ristampa: Nendeln /Liechtenstein/, Kraus, 1973].

JABERG, Karl/Jakob JUD (1987) Atlante linguistico ed etnografico dell'Italia e della Svizzera meridionale. vol. I: L'atlante linguistico come strumento di ricerca. Fondamenti critici e introduzione. Edizione italiana a cura di Glauco Sanga e Serenella Baggio. Milano: Unicopli. 
Kretzschmar, William A./Edgar W. SchneIder (1996) (a cura di) Introduction to Quantitative Analysis of Linguistic Survey Data. An Atlas by Numbers. Thousand Oaks/London/New Delhi: Sage Publications.

Nerbonne, John/William Kretzschmar, Jr. (2006) «Progress in Dialectometry: toward Explanation.» Literary and Linguistic Computing 21/4, 387-397.

Okabe, Atsuyuki/Barry Boots/Kokichi Sugihara (1992) Spatial Tesselations. Concepts and Applications of Voronoi Diagrams. Chichester/New York/Brisbane/Toronto/Singapore: Wiley.

Petracco Siccardi, Giulia (1963) «Influenze genovesi sulle colonie gallo-italiche della Sicilia.» Bollettino del Centro di Studi Filologici e Linguistici Siciliani 9, 106-132.

PfISTER, Max (1988) Galloromanische Sprachkolonien in Italien und Nordspanien. Mainz/Stuttgart: Akademie der Wissenschaften und der Literatur/Steiner.

RoHLFS, Gerhard (1965) «Correnti e strati di romanità in Sicilia (aspetti di geografia linguistica).» Bollettino del Centro di Studi Filologici e Linguistici Siciliani 9, 74-105.

RoHLFs, Gerhard (1975) Historische Sprachschichten im modernen Sizilien. München: Bayerische Akademie der Wissenschaften.

Sneath, Peter H. A./Robert R. Sokal (1971) Numerical Taxonomy. The Principles and Practice of Numerical Classification. San Francisco: Freeman.

Trovato, Salvatore C. (1998) «I dialetti galloitalici della Sicilia.» In: G. Holtus et al. (a cura di), Lexikon der romanistischen Linguistik. Vol. VII: Kontakt, Migration und Kunstsprachen. Kontrastivität, Klassifikation und Typologie. Tübingen: Niemeyer, 538-559.

Trovato, Salvatore C. (a cura di) (1999) Convegno di studi su "Dialetti galloitalici dal Nord al Sud. Realtà e prospettive». Piazza Armerina, 7-9 aprile 1994. Enna: Il Lunario.

WAGNer, Max Leopold (1950) La lingua sarda. Bern: Francke. 



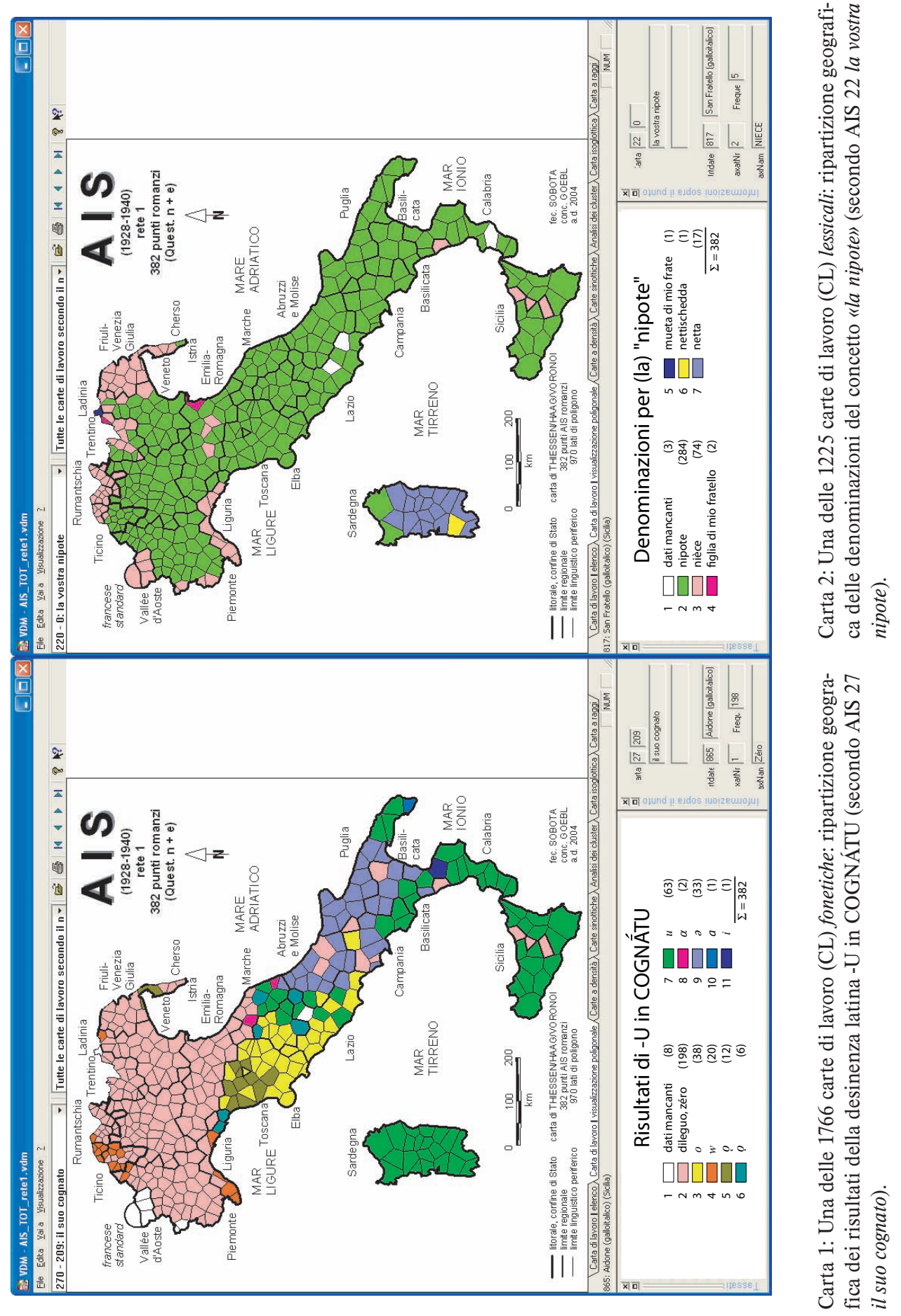



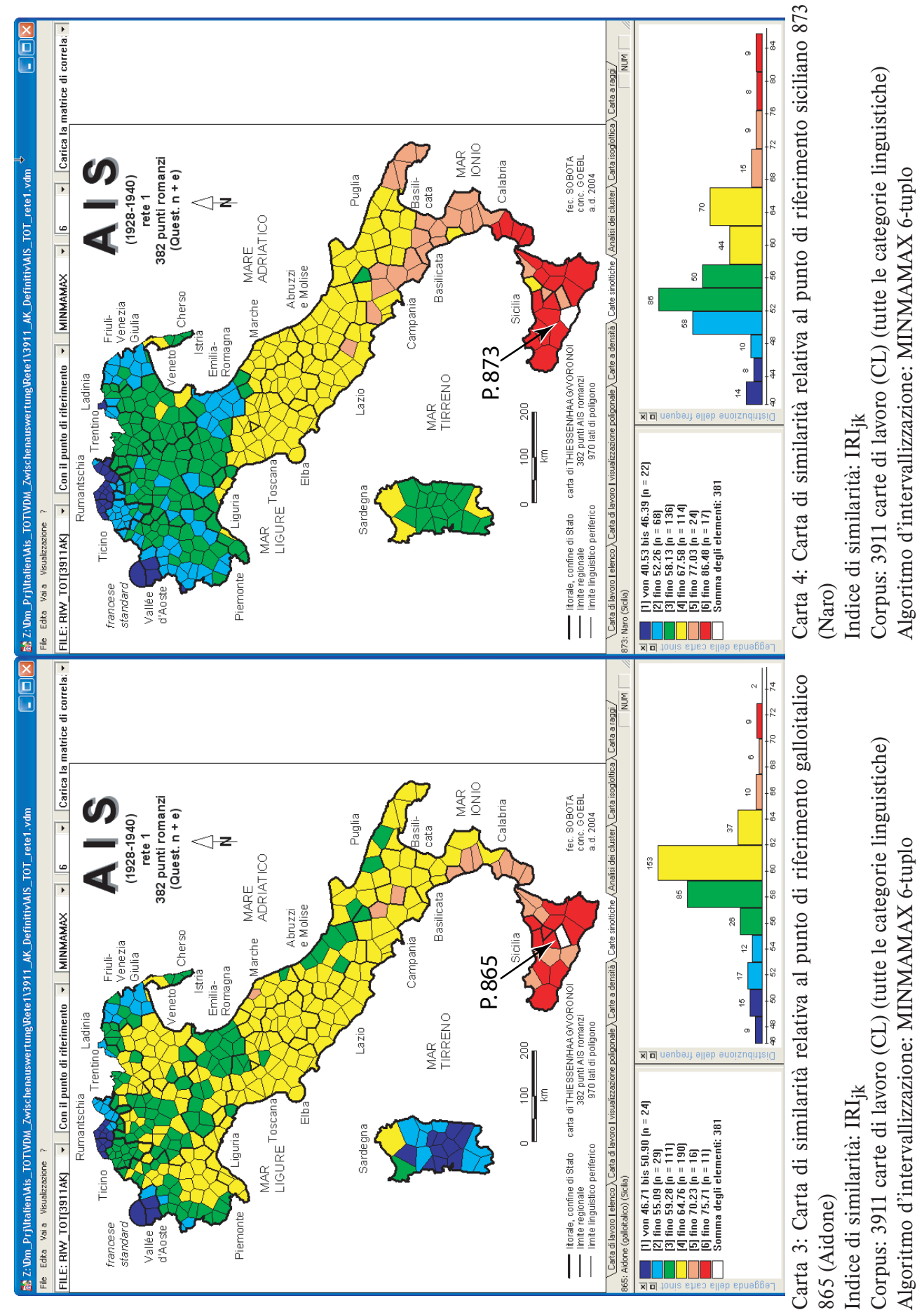

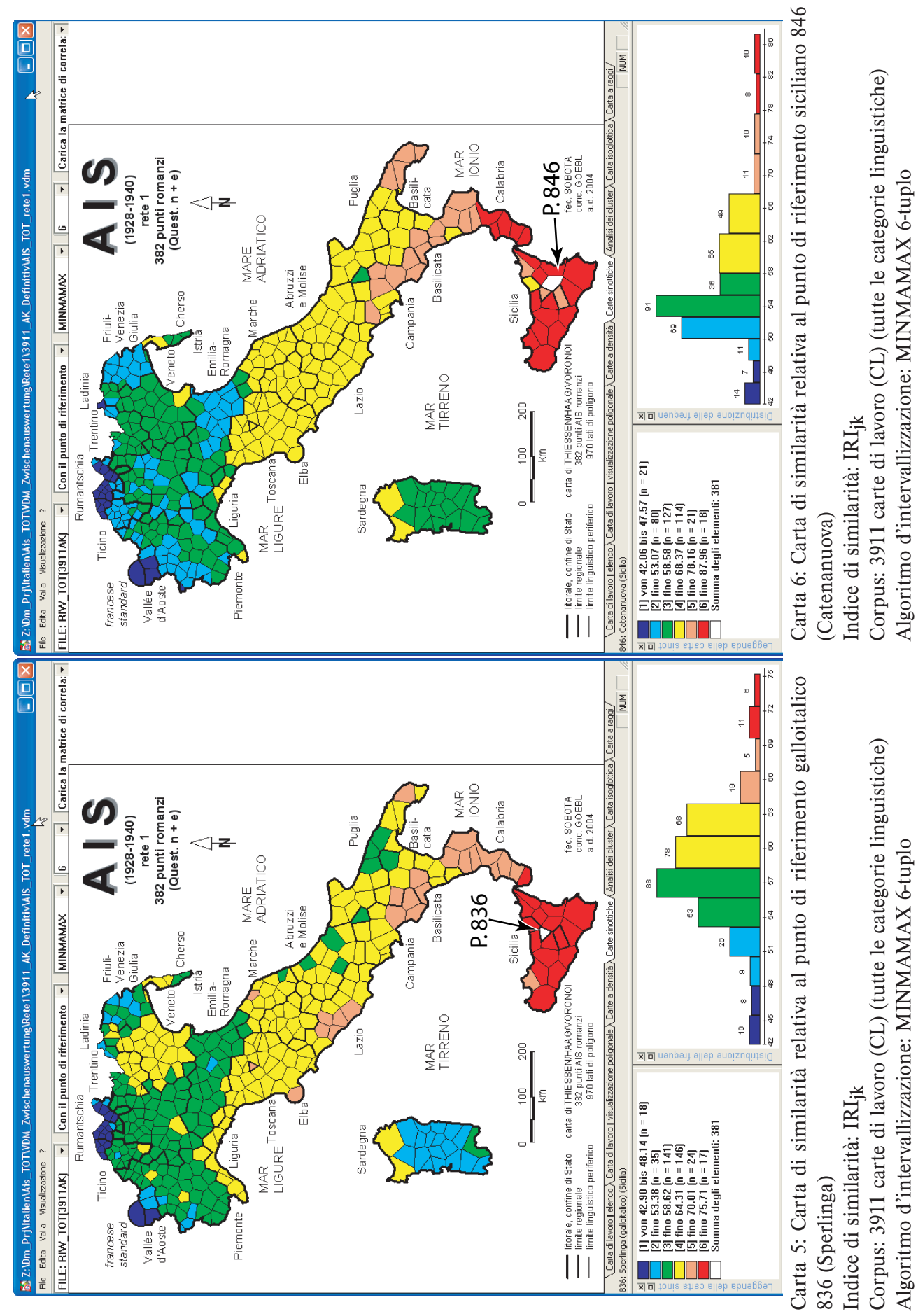


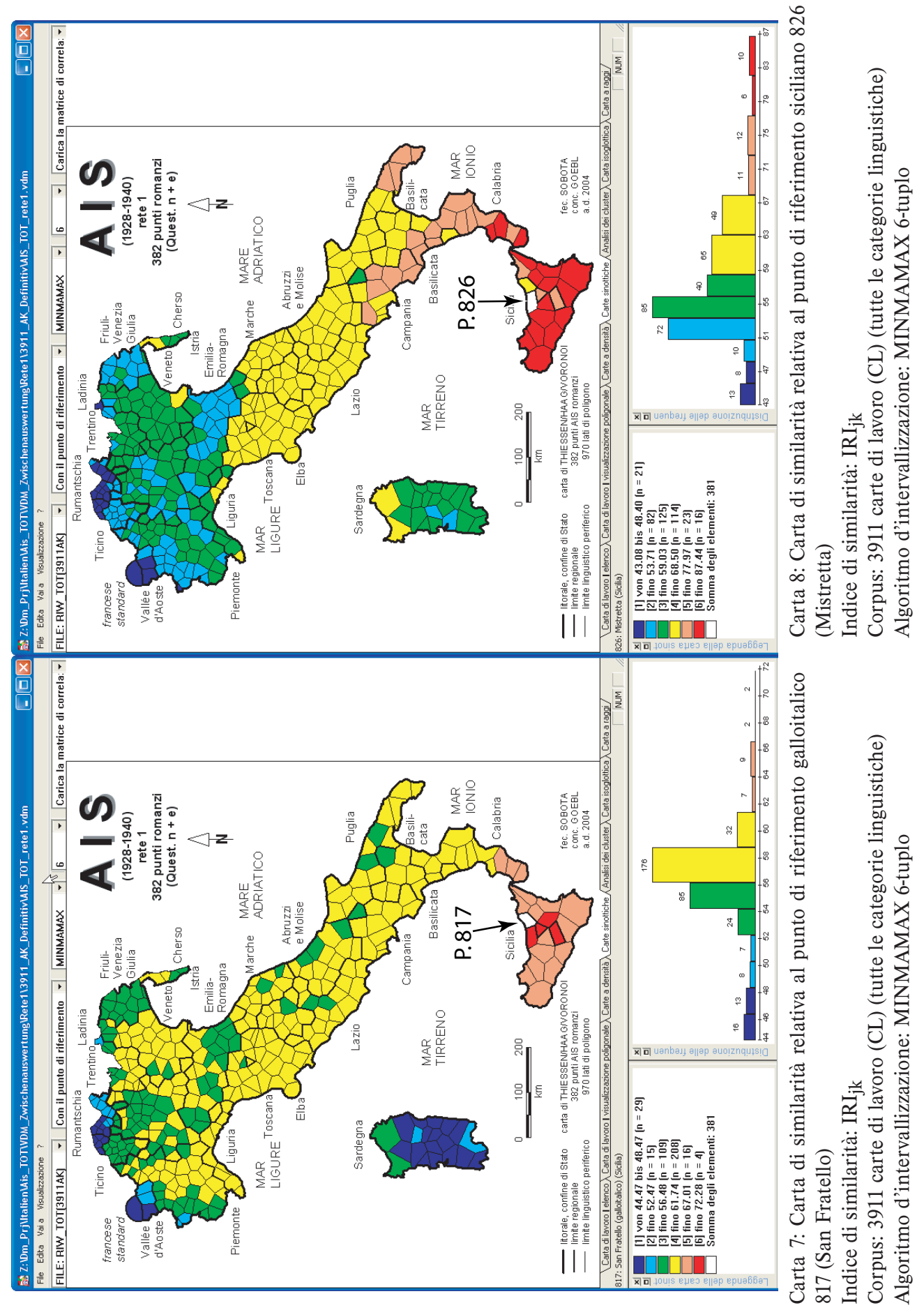




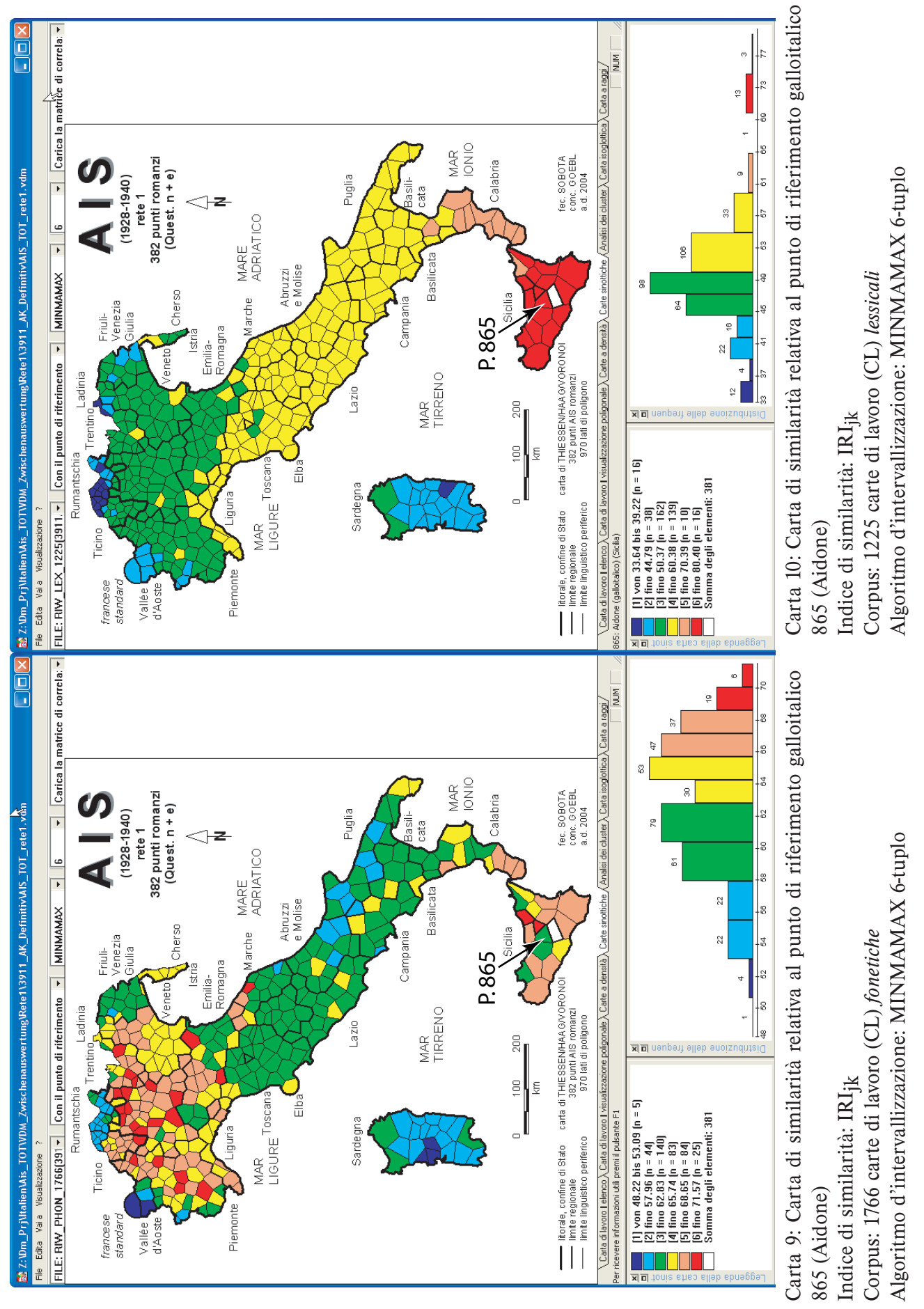




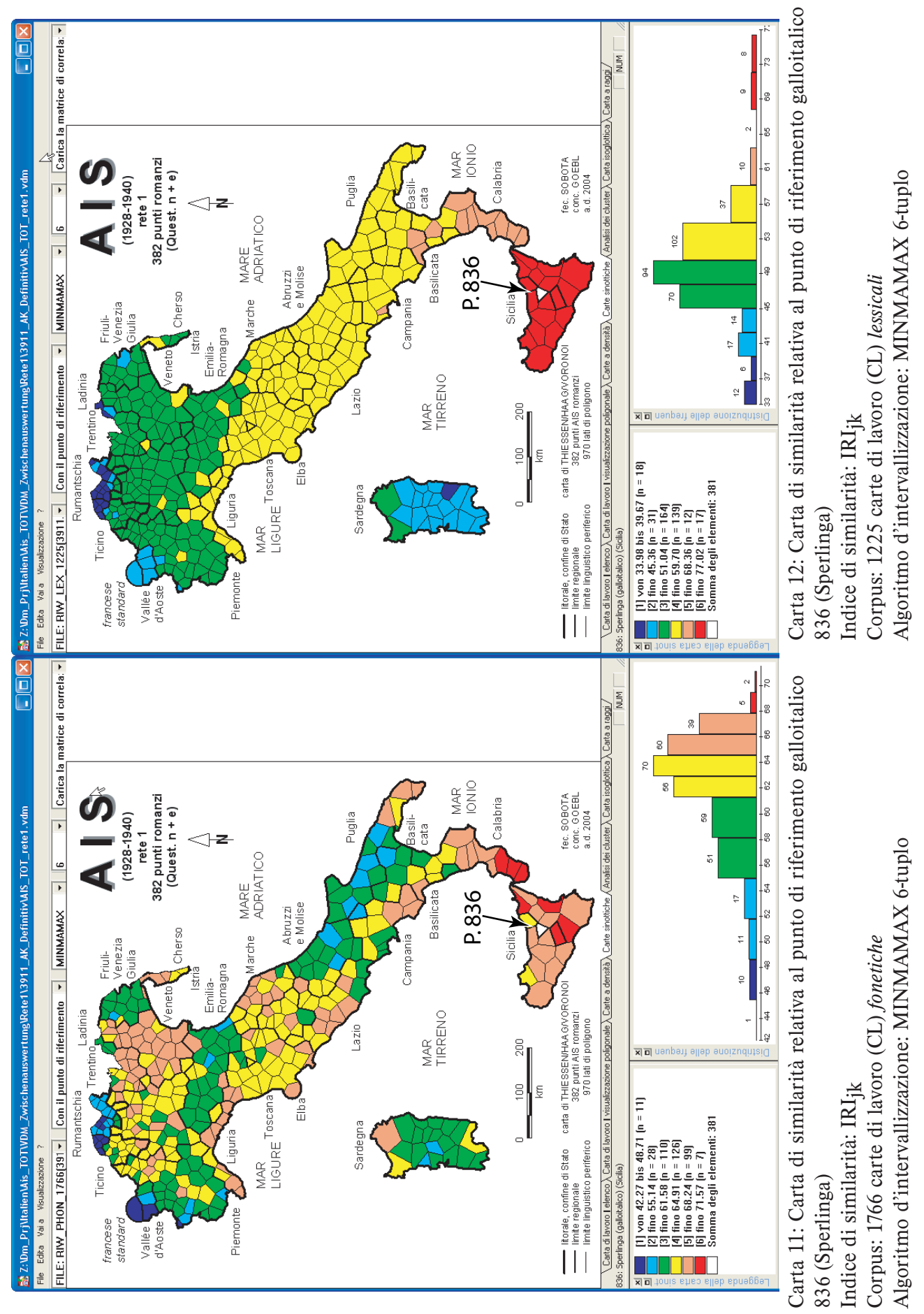




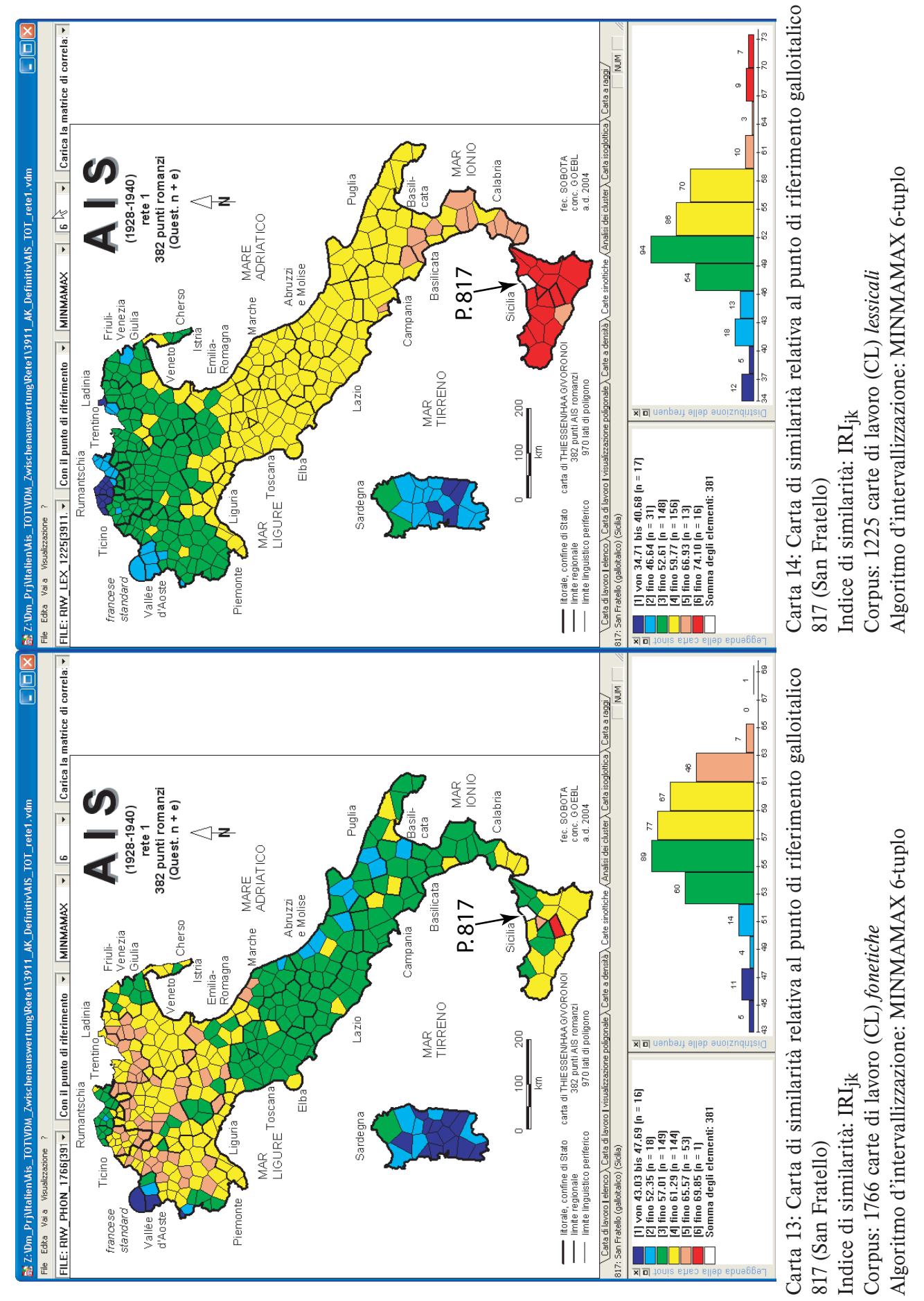




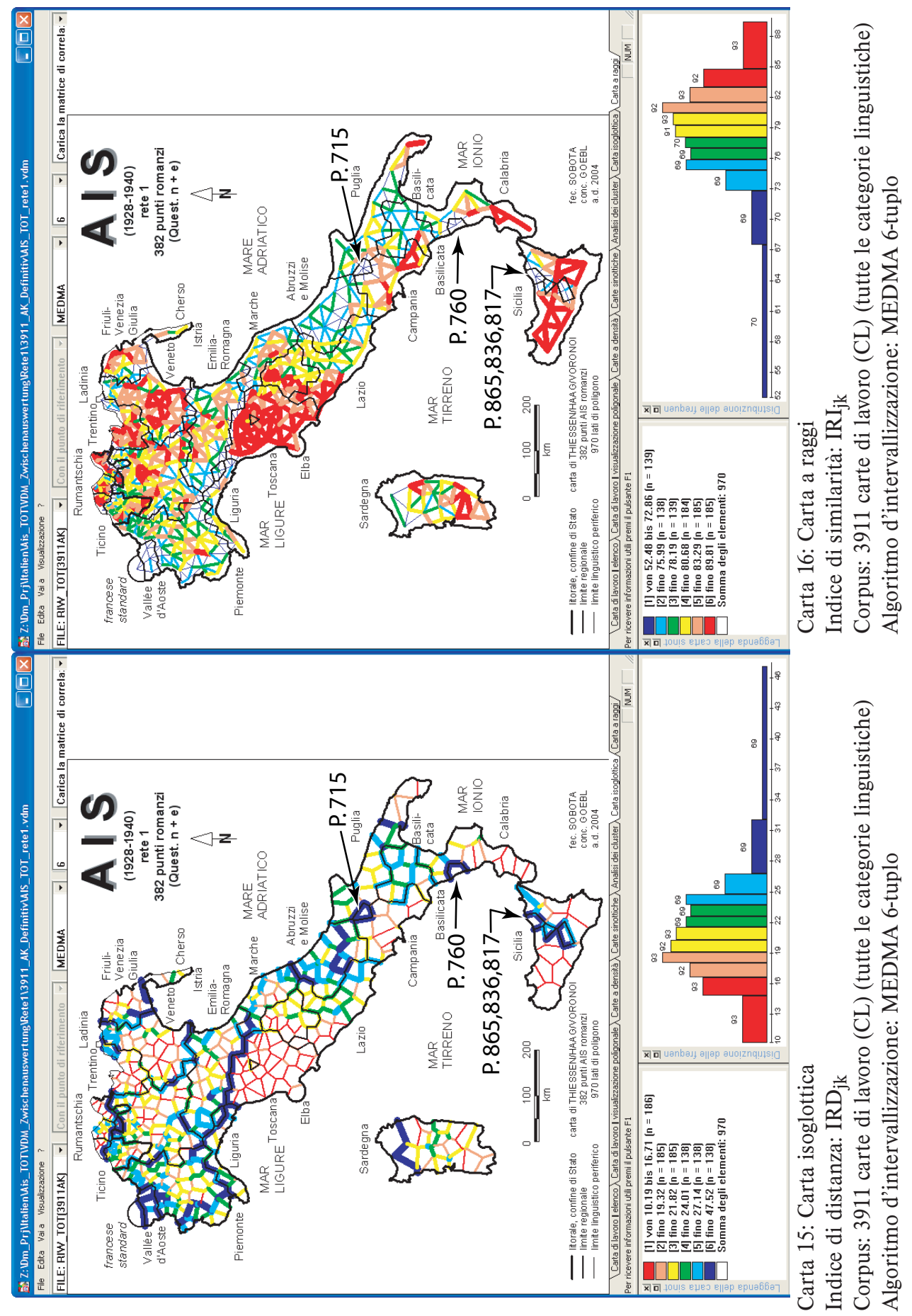




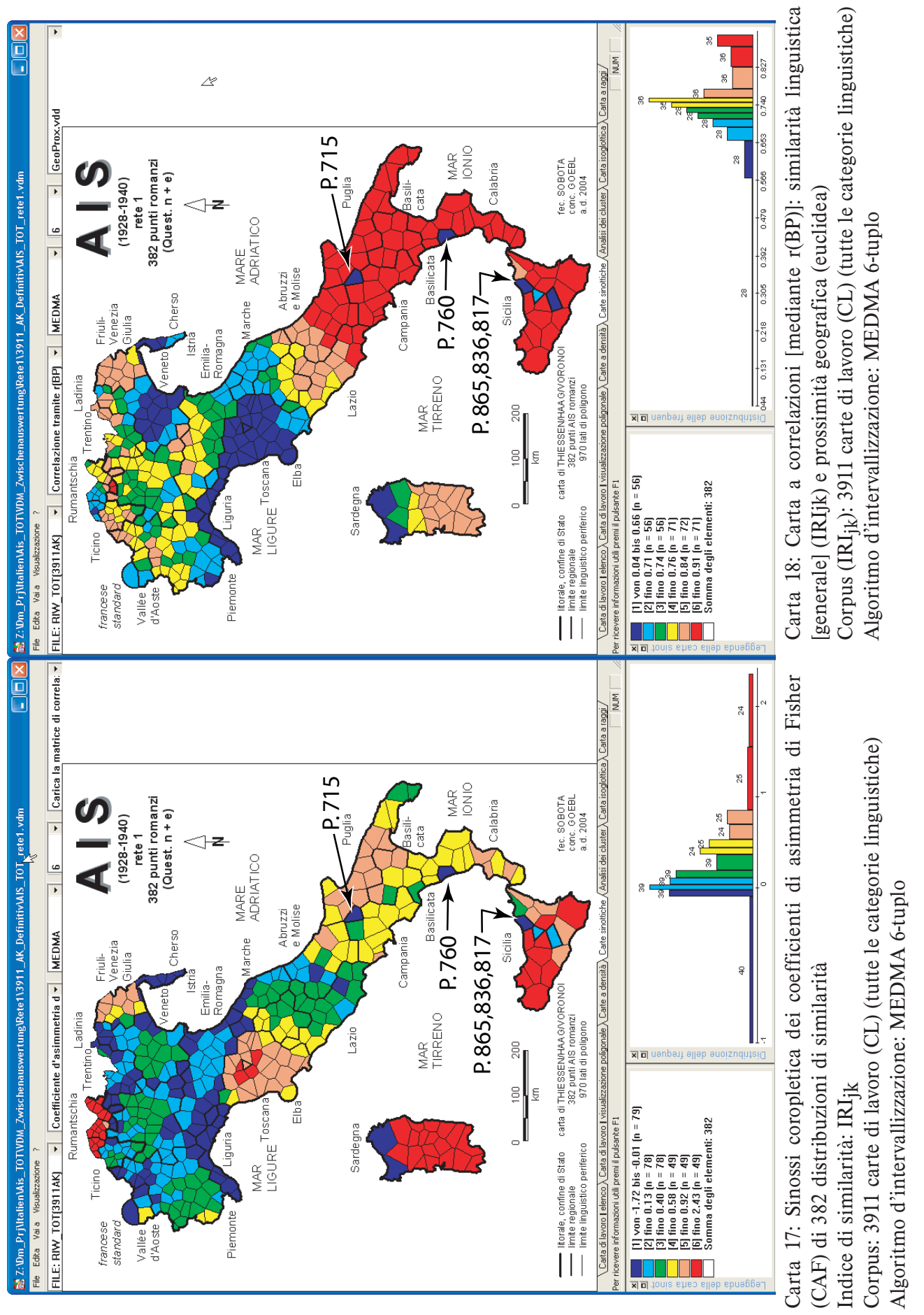




\section{Riassunto \\ DEL POSTO DIALETTOMETRICO CHE SPETTA AI PUNTI GALLOITALICI AIDONE, SPERLINGA E SAN FRATELLO NEL SISTEMA DELLA RETE DELL'AIS}

La rete dell'atlante italo-svizzero AIS comprende sette isole linguistiche tra cui tre sono di natura alloglotta (relative cioè al greco ed all'albanese) mentre cinque sono di origine romanza. In tre delle isole linguistiche romanze, tutte ubicate in Sicilia, si parlano ancora dialetti galloitalici: si tratta delle località di Aidone (P.-AIS 865), San Fratello (P.-AIS 817) e Sperlinga (P.-AIS 836). È ben risaputo che l'origine storica di queste isole risale all'Alto Medioevo (XII-XIII secoli) e che la patria dei rispettivi coloni alto-medievali si trovava nella zona di contatto situata tra il Piemonte meridionale, la Liguria settentrionale e la contigua Emilia occidentale.

Nel corso della dialettometrizzazione della totalità dei dati dell'AIS - svoltasi a Salisburgo negli anni 2005-2009 - si è presentata l'opportunità di esaminare, tramite tutti gli strumenti dialettometrici attualmente a disposizione, tanto la posizione (quantitativa relazionale) dei dialetti galloitalici delle suddette tre località nella rete dell'AIS, quanto quella di alcuni dialetti siciliani limitrofi.

I risultati delle rispettive analisi sono molto sfumati e dimostrano chiaramente la complessità dell'inserzione delle parlate galloitaliche nel diasistema siciliano: vengono presentati tramite 16 carte dialettometriche a colore, ciascuna delle quali è accompagnata di appositi spiegazioni e commenti dialettometrici e linguistici.

\section{Povzetek}

O DIALEKTOMETRIČNEM MESTU, KI PRIPADA GALOITALSKIM TOČKAM AIDONE, SPERLINGA IN SAN FRATELLO V SISTEMU MREŽE AIS

Mreža italijansko-švicarskega atlasa AIS zajema sedem jezikovnih otokov, od katerih so trije neromanski (zadevajo namreč grščino in albanščino), pet pa jih je romanskih. V treh izmed romanskih jezikovnih otokov, ki so vsi na Siciliji, se še vedno govorijo galoitalska narečja: gre za točke Aidone (P.-AIS 865), San Fratello (P.-AIs 817) in Sperlinga (P.-AIS 836). Znano je, da so ti otoki nastali v visokem srednjem veku (12. - 13. stoletje) in da je bila domovina teh kolonov na območju, kjer se stikajo južni Piemont, severna Ligurija in zahodna Emilija.

Med dialektometrizacijo celotne baze podatkov AIS-a - opravljena je bila v Salzburgu v letih 2005-2009 - je bilo mogoče s pomočjo vseh dialektometričnih orodij, ki so trenutno na voljo, proučiti oz. izmeriti tako položaj galoitalskih narečij v omenjenih treh točkah mreže AIS kot položaj nekaterih sicilijanskih narečij, ki so z galoitalskimi narečji v stiku.

Rezultati teh raziskav so zelo niansirani in jasno kažejo, kako kompleksen je odnos med galoitalskimi govori in preostalim sicilijanskim diasistemom; predstavljeni so s pomočjo 16 barvnih dialektometričnih kart, vsaka izmed katerih je opremljena z ustreznimi razlagami ter dialektometričnimi in jezikoslovnimi komentarji. 